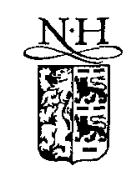

\title{
Field theory questions for string theory answers *
}

\author{
Ofer Aharony $^{a, 1}$, Jacob Sonnenschein ${ }^{b, 2}$, Stefan Theisen ${ }^{c, d, 3}$ \\ Shimon Yankielowicz ${ }^{\text {b,4 }}$, \\ a Department of Physics and Astronomy, Rutgers University, Piscataway, NJ 08855-0849, USA \\ b School of Physics and Astronomy, Beverly and Raymond Sackler Faculty of Exact Sciences, \\ Tel-Aviv University, Ramat-Aviv, Tel-Aviv 69978, Israel \\ c Sektion Physik, University of Munich, Munich, Germany ${ }^{5}$ \\ d. CERN, Theory Division, Geneva, Switzerland
}

Received 13 December 1996; accepted 27 January 1997

\begin{abstract}
We discuss the field theory of 3-brane probes in F-theory compactifications in two configurations, generalizing the work of Sen and of Banks, Douglas and Seiberg. One configuration involves several parallel 3-brane probes in F-theory compactified on $\mathbf{T}^{4} / \mathbb{Z}_{2}$, while the other involves a compactification of F-theory on $\mathbf{T}^{6} / \mathbb{Z}_{2} \times \mathbb{Z}_{2}$ (which includes intersecting $D_{4}$ singularities). In both cases string theory provides simple pictures of the space-time theory, whose implications for the 3-brane world-volume theories are discussed. In the second case the field theory on the probe is an unusual $N=1$ superconformal theory, with exact electric-magnetic duality. Several open questions remain concerning the description of this theory. (C) 1997 Published by Elsevier Science B.V.
\end{abstract}

PACS: $11.25 ; 11.30 \mathrm{P}$

Keywords: String theory; F-theory; D-branes

\section{Introduction}

The study of brane probes in string theory vacua enables us to connect, in a relatively simple way, results in field theory with results in string theory. The first such example involving a four-dimensional field theory was described in $[1,2]$. Sen showed $[1]$ that

\footnotetext{
* Work supported in part by the US-Israel Binational Science Foundation, by GIF, the German-Israeli Foundation for Scientific Research, and by the Israel Academy of Science.

${ }^{1}$ E-mail: oferah@physics.rutgers.edu

${ }^{2}$ E-mail: cobi@ccsg.tau.ac.il

${ }^{3}$ E-mail: theisen@mppmu.mpg.de

${ }^{4}$ E-mail: shimonya@ccsg.tau.ac.il

${ }^{5}$ Permanent address.
} 
an orbifold limit of F-theory [3] on K3 was $T$-dual to type I compactified on $\mathbf{T}^{2}$, and that F-theory provided a consistent description of the smoothing of the orientifold points when moving away from the orientifold limit. The mathematical description of the region (both in space-time and in moduli space) around an orientifold point was found to be exactly the same as Seiberg and Witten's description of the quantum-corrected moduli space of the $S U(2) N=2$ gauge theory with $N_{f}=4$ [4,5]. Banks, Douglas and Seiberg [2] later showed that this similarity was not a coincidence. It arises just by looking at the effective field theory on the world-volume of a 3-brane moving in this vacuum, and demanding consistency between the quantum corrections in this field theory and in the space-time theory (whose fields couple to the 3-brane world-volume). This sort of relation (first discussed in [6]) should presumably hold for any D-brane moving in a string theory vacuum, as long as the probe does not modify the vacuum in a substantial way. It provides a general relation between string theory, and its low-energy effective space-time theories, and the world-volume field theories of D-branes. The D-branes thus may serve as probes which enable us to learn more about non-perturbative aspects of string theory (and of field theory). When the resolution of the singularities in string theory is known, we can use it to learn about the resolution of singularities in field theory, and vice versa. This approach has since been generalized to several other cases (for instance, in Refs. [7-10]).

In this note we wish to analyze two generalizations of this approach. First, in Section 2, we discuss the field theory of multiple parallel D-branes as probes, in the same string theory vacuum considered in $[1,2]$. In Section 3 we analyze a generalization to six dimensions, discussing F-theory on $\mathbf{T}^{6} / \mathbb{Z}_{2} \times \mathbb{Z}_{2}$ (or, more precisely, an orientifold which differs from this by discrete torsion). In both cases the string theory (F-theory) "answer" is trivially known, and we are looking for the classical field theory on the 3-brane which reproduces this "answer" when computing its quantum moduli space. In the first case this field theory turns out to be a simple $N=2$ field theory, for which it is easy to see also directly in field theory that the moduli space decomposes into several copies of the Seiberg-Witten moduli space. In the second case we find an $N=1$ superconformal field theory which does not seem to be known, and which has many unusual properties, including an exact electric-magnetic duality. We compute the field content of this theory, but we do not know how to write down an explicit superpotential to describe it. In Section 4 we give a summary of our results and of the remaining open questions. In Appendix A we describe explicitly our calculations of the field content of the 3-brane probe theory near an orientifold point in the string theory discussed in Section 3. As this paper was being completed, a preprint by Sen [11] appeared, in which he treats a different orientifold, namely a $T$-dual version of the Gimon-Polchinski [12] model. The analogous analysis for this model, which leads to a different space-time and also world-volume theory, can be easily carried through and one finds that the problems we have encountered with our original model are absent there. We have included a brief discussion of this model, along the lines exposed in Section 3, at the end of Section 4. Further details of this model, in particular the space-time aspects and its non-perturbative description, are found in [11]. 


\section{Multiple 3-branes as probes in eight dimensions}

In this section we analyze a trivial generalization of the approach of Banks, Douglas and Seiberg [2], which involves using $k$ 3-branes as probes instead of a single 3-brane. Of course, since there are no forces between parallel 3-branes [13] (and we will use only parallel 3-branes), we expect the result to be the same as the result found by Sen. Each 3-brane should see one region of the same vacuum of the string theory, according to its space-time position, and the moduli space should be the product of the moduli spaces of the different 3-branes (up to an $S_{k}$ "Weyl group" which permutes the identical 3-branes). This expectation will be confirmed by the field theory analysis of the world-volume theory on the 3-branes, as we will now show.

As in [2], we will begin with the description of this theory as type I on $\mathbf{T}^{2}$. Instead of looking at a single 5-brane wrapped around $\mathbf{T}^{2}$, we will now take $k$-branes wrapped around $\mathbf{T}^{2}$. When the 5-branes are at the same position, the field theory describing them is well-known (see, for example, Ref. [14]). There is an $S p(2 k)$ gauge group, ${ }^{6}$ with a hypermultiplet in the antisymmetric representation (which decomposes into an irreducible $\mathbf{k}(2 \mathbf{k}-\mathbf{1})-\mathbf{1}$ and a singlet). This is an $N=1$ theory in $5+1$ dimensions, which becomes an $N=2$ theory in $3+1$ dimensions when we reduce it on the torus. The Wilson loops of the gauge field around the two compact dimensions become expectation values of the two scalar fields of the $N=2$ vector multiplets in four dimensions. The scalars in the antisymmetric representation hypermultiplet describe the motion of the 3-branes in the four non-compact transverse dimensions. In the 10D type I theory there are 32 additional half-hypermultiplets in the $2 \mathbf{k}$ representation of the $S p(2 k)$ gauge group (and in the fundamental representation of the $S O(32)$ space-time gauge symmetry). As discussed in [2], due to an interplay between the $S O(32)$ Wilson loops and the $S p(2 k)$ Wilson loops, four of these hypermultiplets are light when the probe is near an orientifold point. In the F-theory these arise from strings connecting the 3-brane probe with the four 7-branes which sit at each orientifold point. As in Ref. [1], we take the torus $\mathbf{T}^{2}$ to be very large and analyze only the region near a single orientifold point, when all fields corresponding to the other points are very massive.

To summarize, the world-volume field theory corresponding to $k$ parallel 3-branes moving in this vacuum is an $\operatorname{Sp}(2 k) N=2$ gauge theory, with four fundamental hypermultiplets and an additional hypermultiplet in the antisymmetric representation. The beta function of this theory is zero, as required by the string theory picture which has no scale (when the 3-brane is exactly at the orientifold point). The theory has an $S O(8)$ global symmetry acting on the "quark" hypermultiplets, which corresponds to the $S O(8)$ gauge symmetry in space-time. The vector multiplet in the 7+1-dimensional space-time includes also a complex scalar field in the antisymmetric (adjoint) 28 representation of $S O(8)$. In the world-volume field theory, this corresponds to a mass parameter for the quarks in the $\mathbf{2 k}$ representation. In the F-theory picture these mass parameters correspond

\footnotetext{
${ }^{6}$ In this paper we denote by $S p(2 k)$ the symplectic group whose fundamental representation is of dimension $2 k$.
} 
to the motion of the 7-branes in the two compact directions. These are the only scalar fields in space-time, so they are the only parameters we have in our world-volume theory. In the field theory one could also add a mass term for the antisymmetric hypermultiplet, which would prevent the 3 -branes from moving in the four non-compact directions. It would be very interesting to see whether such a mass deformation can be accounted for in string theory (so far no string theory realization of such a term is known).

Next, we should analyze the quantum moduli space of this field theory. As in [2], we will analyze only the Coulomb phase of the theory (the Higgs phase should presumably be related to $S O(8)$ instantons in space-time). In this phase, the scalar field in the vector multiplet has an arbitrary expectation value, which may be brought (by gauge transformations) to a diagonal matrix of the form $\operatorname{diag}\left(a_{1}, a_{2}, \ldots, a_{k},-a_{1},-a_{2}, \ldots,-a_{k}\right)$, where the $a_{i}$ are arbitrary complex numbers. This vev breaks the $\operatorname{Sp}(2 k)$ symmetry to $U(1)^{k}$. The eigenvalues $a_{i}$ correspond (when squared) to the position of the $k 3$-branes in the 2 compact coordinates, measured from the orientifold point. When we are at an arbitrary point in the Coulomb phase, the superpotential (whose form is dictated by $N=2$ supersymmetry) does not allow an expectation value for the hypermultiplets in the fundamental representation. However, the flat directions corresponding to the hypermultiplet in the antisymmetric representation are not all lifted by the adjoint vev, and a moduli space of $k$ dimensions remains flat (one of these is the singlet component of the antisymmetric representation, while the other $k-1$ are part of the $\mathbf{k}(2 \mathbf{k}-\mathbf{1})-\mathbf{1}$ representation). This corresponds to a vev for the antisymmetric matrix of the form

$$
\left(\begin{array}{cccccccc}
0 & 0 & \ldots & 0 & b_{1} & 0 & \ldots & 0 \\
0 & 0 & \ldots & 0 & 0 & b_{2} & \ldots & 0 \\
\vdots & \vdots & \ddots & \vdots & \vdots & \vdots & \vdots & \vdots \\
0 & 0 & \ldots & 0 & 0 & 0 & \ldots & b_{k} \\
-b_{1} & 0 & \ldots & 0 & 0 & 0 & \ldots & 0 \\
0 & -b_{2} & \ldots & 0 & 0 & 0 & \ldots & 0 \\
\vdots & \vdots & \vdots & \vdots & \vdots & \vdots & \ddots & \vdots \\
0 & 0 & \ldots & -b_{k} & 0 & 0 & \ldots & 0
\end{array}\right)
$$

Each of the four scalars in the antisymmetric hypermultiplet has an independent vev, and they all commute so that all of them can be brought to the form (2.1) simultaneously. The simplest way to consider this is to treat the $b_{i}$ above as quaternionic numbers. In the absence of the adjoint vev, the vev (2.1) breaks the $S p(2 k)$ symmetry to $S U(2)^{k}$. The $b_{i}$ correspond to the position of the 3-branes in the non-compact transverse dimensions ( $x_{4}, x_{5}, x_{6}$ and $x_{7}$ ). However, in $N=2$ supersymmetric theories, the moduli space decomposes into the product of the vector multiplet moduli space and the hypermultiplet moduli space. Thus, the vector multiplet moduli space cannot depend on the scalars $b_{i}$ (which can get vevs at arbitrary points in the Coulomb phase). In particular, the vector multiplet moduli space remains the same if we take all the $b_{i}$ to be very large, flowing to an $S U(2)^{k}$ gauge theory. In this limit nothing (but singlets) remains of the antisymmetric hypermultiplet, and we remain with $k$ copies of the Seiberg-Witten theory of $S U(2)$ 
gauge group with $N_{f}=4$. Globally we should divide this by a discrete $S_{k}$ symmetry (which is a subgroup of the Weyl group of $S p(2 k)$ ), since all copies (3-branes) are identical. All copies have the same masses for the quark hypermultiplets (corresponding to the 7-brane positions in the F-theory description), but have independent values of the adjoint vevs (which are the $a_{i}$ above), corresponding to the position of the 3-brane in the compact space. Thus, we have recovered the same picture we expected to find from the string theory point of view, since there are no interactions between the 3-branes. At special points in the moduli space we have enhanced global symmetries, corresponding to enhanced gauge symmetries in space-time. The discussion of these is exactly analogous to the discussion in $[1,2]$.

Now that we understand the moduli space of this theory, we can try to learn from it about other field theories we can flow to. First, let us look at the case of $k=2$, where the gauge group is $S p(4) \sim S O(5)$. From the $S O(5)$ point of view, we have one fundamental hypermultiplet and four spinors (the singlet decouples) and (as discussed above) the moduli space decomposes into two copies of an $S U(2)$ moduli space. Since we can give arbitrary masses to the spinors, the same should also be true for the $S O(5)$ gauge theory with a single fundamental hypermultiplet, which was analyzed in the past. However, by an argument analogous to the one we used above, it is trivial to see that the Coulomb phase of an $S O(2 r+1)$ gauge theory with $N_{f}$ flavors (one of which is massless) is generally the same as the Coulomb phase of an $S O(2 r)$ gauge theory with $N_{f}-1$ flavors. This is because we can give an arbitrary vev to one fundamental hypermultiplet without disturbing the Coulomb phase, and it is corroborated by the explicit form of the curves (as written, for instance, in Ref. [15]).

Another theory we can flow to is the $U(k) N=4$ theory, which we can reach by taking the 3-branes to be together but far away from the orientifold point [16]. In the gauge theory description, we reach this limit by taking the modulus $u \rightarrow \infty$ in all of the $S U(2)$ curves, and we arrive at $k$ copies of the (free) $U(1)$ gauge theory (up to the $S_{k}$ identification). Indeed, this is the correct description of the $U(k) N=4$ theory, as analyzed by Donagi and Witten [17]. We can also show this, as above, by decomposing the $N=4$ vector multiplet into an $N=2$ vector multiplet and hypermultiplet, and giving large vevs to the hypermultiplet (which should not affect the vector moduli space), breaking the gauge symmetry to $U(1)^{k}$.

To summarize, multiple $p$-branes may also be used to learn from field theory about string theory or vice versa. However, in the Coulomb phase of the field theory they just lead to $k$ copies (up to global identifications) of the Coulomb phase of the field theory found for a single $p$-brane. We can show, using the string theory, that the moduli space of these field theories indeed decomposes in this way, but so far in all the examples it is easy to see this also directly from field theory arguments. The superconformal theory at the origin of the moduli space is, however, not trivially related to the theory found for a single 3-brane. ${ }^{7}$ For instance, we can turn on a mass for the antisymmetric hypermultiplet in this theory and flow to an $N=2 S p(2 k)$ gauge theory with $N_{f}=4$, which behaves

\footnotetext{
${ }^{7}$ We thank N. Seiberg for stressing this point.
} 
differently for different values of $k$. Thus, the analysis of multiple brane probes may lead to the discovery of new superconformal field theories, though their Coulomb phases would always be simply related to those of the single probe theory.

\section{3. $N=1$ 4D SCFTs from 3-branes at intersecting singularities}

In F-theory compactifications to eight dimensions, the 3-brane probe has $N=2$ supersymmetry. Interesting $N=2$ SCFTs arise when the 3-brane is brought to a singularity of the compactification manifold. Non-trivial conformal field theories arise when the singularity is of type $A_{0}, A_{1}, A_{2}, D_{4}, E_{6}, E_{7}$ or $E_{8}$. The first four correspond to known $N=2$ SCFTs [18] while the other $N=2$ SCFTs were recently discussed from various points of view in $[19,20,10]$. Going down to six dimensions, there are many more possibilities for singularities in the manifold, and generically the 3-brane field theory has only $N=1$ supersymmetry. In [9], the behavior of a 3-brane probe near an ADE singularity fibered over the additional two compact dimensions [21] was analyzed, leading to several new $N=1$ SCFTs. In this section we discuss the behavior of a 3-brane probe near the intersection of two singularities, which also corresponds to $N=1$ SCFTs.

The only singularity which can occur at a weak (but non-zero) value of the coupling is a $D_{4}$ singularity [5], so this is the only case where we definitely expect to have a lagrangian description of the probe theory. As in [1], let us look for compactifications of F-theory on CY manifolds which have a constant value of $\tau$ (which we can take to correspond to weak coupling). The simplest case in which this occurs is the elliptic fiber over a base $\mathbf{C} \mathbf{P}^{1} \times \mathbf{C} \mathbf{P}^{1}$ [22]. In general such a fibration is described by an equation of the form

$$
y^{2}=x^{3}+f\left(z_{1}, z_{2}\right) x+g\left(z_{1}, z_{2}\right)
$$

where $z_{1}$ and $z_{2}$ label the two $\mathbf{C P}^{1}$ s of the base. The torus of the fiber degenerates where the discriminant $\Delta=4 f^{3}+27 g^{2}$ vanishes, and in F-theory the solutions to $\Delta=0$ are the locations of type IIB 7-branes in the compact dimensions, and $\tau$ has a non-trivial monodromy when going around them. $j\left(\tau\left(z_{1}, z_{2}\right)\right)$ is then proportional to $f^{3} / \Delta$, and we want this to be constant. The simplest choice (though not the most general solution) is of the form

$$
f\left(z_{1}, z_{2}\right)=\alpha \phi_{1}\left(z_{1}\right)^{2} \phi_{2}\left(z_{2}\right)^{2}, \quad g\left(z_{1}, z_{2}\right)=\phi_{1}\left(z_{1}\right)^{3} \phi_{2}\left(z_{2}\right)^{3},
$$

where $\phi_{1}$ and $\phi_{2}$ are general polynomials of degree four:

$$
\phi_{1}\left(z_{1}\right)=\prod_{i=1}^{4}\left(z_{1}-z_{1}^{(i)}\right), \quad \phi_{2}\left(z_{2}\right)=\prod_{i=1}^{4}\left(z_{2}-z_{2}^{(i)}\right) .
$$

It is clear that at this point in moduli space $\tau$ has a constant value depending on $\alpha$, as in [1], and that we have $D_{4}$ singularities at $z_{1}=z_{1}^{(i)}$ and at $z_{2}=z_{2}^{(i)}$. 
In general the space-time field theory at an intersection of two $D_{4}$ singularities is not well understood [21,23]. However, as in Sen's case [1], we can try to interpret this point in moduli space as an orientifold of the type IIB theory, and since it can be weakly coupled we can analyze the space-time field theory by perturbative string theory methods. Around each of the points $z_{1}=z_{1}^{(i)}$ (for constant $z_{2}$ ) we have an $S L(2, \mathbb{Z})$ monodromy of

$$
\left(\begin{array}{cc}
-1 & 0 \\
0 & -1
\end{array}\right) \text {. }
$$

Locally, we can interpret this as an orientifold of the type IIB theory by $(-1)^{F_{Z}} \cdot \Omega$, as discussed in [1], which should be accompanied by four 7-branes to cancel the RR charge. Each point $z_{1}=z_{1}^{(i)}$ on the first $\mathbf{C P}^{1}$ factor carries a deficit angle of $\pi$, all four of them together deforming the $\mathbf{C} \mathbf{P}^{1}$ to $\mathbf{T}^{2} / \mathbb{Z}_{2}$. The same is true at the points $z_{2}=z_{2}^{(i)}$, and altogether it seems that we can write this theory as the type IIB theory on $\mathbf{T}^{4}$, divided by a product of two $\mathbb{Z}_{2}$ symmetries of the form found by Sen [1], one of which inverts $z_{1}$ while the other inverts $z_{2}$. This corresponds to the orientifold of F-theory on $\mathbf{T}^{6}$ by $\left(\mathbb{Z}_{2}\right)^{2}$, discussed in [24-26], which can be deformed into the $(51,3) \mathrm{CY}$ manifold (by changing the values of tensor-multiplet scalars).

However, we should be careful in identifying this orientifold compactification directly with the F-theory compactification described by (3.1)-(3.3). Generally, in F-theory compactifications, the 2-form tensor fields of type IIB are assumed to vanish. This is, of course, consistent with the $S L(2, \mathbb{Z})$ monodromies that these fields have around singularities of the elliptic fibration. However, at the intersection of two $D_{4}$ singularities that we have been discussing, there is the possibility of blowing up the intersection point to get an additional 2-cycle [21,23]. When there are vanishing 2-cycles, the possibility arises of having discrete 2 -form fields concentrated on the vanishing 2 -cycle, a phenomenon which is related in string theory to discrete torsion [27]. In the naive F-theory compactification, we assume that these fields are zero, and then one can wrap a 3-brane around the vanishing 2-cycle, giving rise to tensionless strings living on the intersection of the $\mathrm{D}_{4}$ singularities. Our orientifold construction, on the other hand, involves a well-defined weakly coupled conformal field theory, whose low-energy spectrum is well-defined and does not include such tensionless strings. ${ }^{8}$ Thus, we conclude that the orientifold differs from the F-theory construction by a discrete 2-form field, which prevents the 3-brane from wrapping around the vanishing 2-cycle (a similar phenomenon involving wrapped 2-branes was described in [28]). Discrete $\mathbb{Z}_{2}$ symmetries force the value of both 2-form tensor fields (integrated over the vanishing 2-cycle) to be either 0 or $1 / 2$ (modulo 1 ). A non-zero value for either (or both) of these fields breaks the $S L(2, \mathbb{Z})$ symmetry to a discrete $\Gamma(2)$ subgroup. For instance, if both fields equal $1 / 2$, this subgroup is generated by $S$ and by $T^{2}$ (where $S$ and $T$ are the standard $S L(2, \mathbb{Z})$ generators; note that in the cases we are discussing the string coupling $\tau$ equals $\tau=\frac{8 \pi i}{g^{2}}+\frac{\theta}{\pi}$ in the field theory, so only $T^{2}$ is naively guaranteed to be a symmetry, as in [5]). The other cases are related

\footnotetext{
${ }^{8}$ We thank E. Witten for emphasizing this point.
} 
to this by $S L(2, \mathbb{Z})$ transformations. In the remainder of this section, we will discuss a 3-brane probe in the orientifold background, which is thus expected to describe an $N=1$ SCFT with a $\Gamma(2)$ electric-magnetic duality symmetry. At the end of the section we will comment on the relation between this theory and the theory of a probe in the F-theory background with no 2-form fields turned on.

The space-time theory corresponding to the orientifold is an $N=1$ supersymmetric theory, whose field content was computed in [24-26]. The untwisted closed string sectors give rise to a supergravity multiplet, to one tensor multiplet and to four hypermultiplets. The open string sectors give an $S O(8)$ gauge group for each group of 7-branes (i.e. each fixed point of one of the $\mathbb{Z}_{2}$ 's), for a total gauge symmetry of $S O(8)^{8}$. No massless states arise from strings between the different groups of 7-branes, but the twisted closed string sector gives rise to a tensor multiplet at each intersection of fixed points (i.e. at each fixed point of both $\mathbb{Z}_{2}$ 's), accounting for a total of 16 additional tensor multiplets. The space-time theory satisfies all of the anomaly cancelation requirements. At each intersection of $\mathrm{D}_{4}$ singularities there is a possibility of blowing up a point to get an additional 2-cycle (by turning on the scalar in the corresponding tensor multiplet). After all these blow-ups we get F-theory on a smooth $(51,3)$ Calabi-Yau manifold. Note that the space-time theory we found (using the orientifold construction) is not the same as the theory we supposedly started with, which was F-theory compactified on an elliptic fibration over $\mathbf{C P}^{1} \times \mathbf{C P}^{1}$. It may be possible to reach that theory by performing phase transitions which turn the 16 additional tensor multiplets we have into hypermultiplets, but we cannot discuss this directly in the orientifold construction.

We would like to analyze what happens to a 3-brane probe as it moves around in this string theory vacuum. Near each fixed line (i.e. a fixed point of one of the two $\mathbb{Z}_{2}$ 's), the space-time theory is the same as the one analyzed in $[1,2]$ and in the previous section. Thus, the theory on the probe should be just an $N=2 S U(2)$ gauge theory with four quark hypermultiplets. Things get more interesting if we move the probe to the intersection of two of the fixed lines (which we will take to be at $z_{1}=z_{2}=0$ ). We expect the probe theory there to have only $N=1$ supersymmetry, since the 7-branes intersect transversely at this point and each breaks a different half of the world-volume $N=4$ supersymmetry. The probe theory should have two deformations, corresponding to turning on $z_{1}$ or $z_{2}$, which should cause it to flow to the $N=2 S U(2)$ theory.

In general, it is difficult to analyze the world-volume theory of a 3-brane probe in F-theory vacua when it is adjacent to 7-branes of different types (which are not just D7-branes). However, since in this case we have an orientifold description of this vacuum, we can use it to compute the states living on the 3-brane. In this way we can compute the states on the 3-brane arising from fundamental strings, which will lead to electrically charged states on the 3-brane. Since the theory on the probe is manifestly invariant under a $\Gamma(2)$ electric-magnetic duality symmetry, we expect that there will also be $(p, q)$ strings which give rise to $(p, q)$ dyonic states in the world-volume theory. However, since our theory has only $N=1$ supersymmetry and no BPS formulas, it is not obvious that these states should remain stable also at weak electric coupling (unlike the $N=2$ case). 
Let us begin by computing the fields corresponding to strings which stretch out from a 3-brane and fold back to the same brane. The 3-brane has three images under $\mathbb{Z}_{2} \times \mathbb{Z}_{2}$, so that every open string state is enhanced to a $4 \times 4$ matrix (as in Ref. [12]), on which we should impose the orientifold restrictions. The $\gamma$ matrices (in the notation of [12]) corresponding to each of the generators in the $\mathbb{Z}_{2} \times \mathbb{Z}_{2}$ may be chosen to be

$$
\gamma_{\Omega_{1}}=\left(\begin{array}{cccc}
0 & i & 0 & 0 \\
-i & 0 & 0 & 0 \\
0 & 0 & 0 & i \\
0 & 0 & -i & 0
\end{array}\right), \quad \gamma_{\Omega_{2}}=\left(\begin{array}{cccc}
0 & 0 & i & 0 \\
0 & 0 & 0 & i \\
-i & 0 & 0 & 0 \\
0 & -i & 0 & 0
\end{array}\right)
$$

and the orbifold matrix is then necessarily

$$
\gamma_{T}=\gamma_{\Omega_{1}} \gamma_{\Omega_{2}}=\left(\begin{array}{cccc}
0 & 0 & 0 & -1 \\
0 & 0 & 1 & 0 \\
0 & 1 & 0 & 0 \\
-1 & 0 & 0 & 0
\end{array}\right)
$$

These matrices are hermitian and unitary, and they correctly reflect the two $\mathbb{Z}_{2}$ actions on the compact coordinates $\left(z_{1}, z_{2}\right)$.

The wave-function matrices of states, $M_{i, j}$, must then satisfy [12] conditions of the type

$$
\begin{aligned}
& M= \pm \gamma_{\Omega_{1}} M^{T} \gamma_{\Omega_{1}}^{-1}, \\
& M= \pm \gamma_{\Omega_{2}} M^{T} \gamma_{\Omega_{2}}^{-1} \\
& M= \pm \gamma_{T} M \gamma_{T}^{-1}
\end{aligned}
$$

where the signs are determined by the transformation properties of the relevant state. For the gauge fields they are,,--+ , for the chiral superfield $X_{6,7}$ they are,,-+- , for $X_{8,9}$ they are,,+-- and for $X_{4,5}$ they are,,+++ .

Performing this computation, details of which can be found in Appendix A, we find that the gauge fields on the 3-brane world-volume give rise to six states, which generate an $S U(2) \times S U(2)$ algebra. The fields $X_{6,7}$ and $X_{8,9}$ each give rise to four states, which are in the $(\mathbf{2}, 2)$ representation of the gauge group, and which are in $N=1$ chiral multiplets which we will denote by $A$ and $B$. As in [2], we will identify the gauge singlet $A^{2}$ with the $z_{1}$ coordinate of the 3 -brane, and $B^{2}$ with the $z_{2}$ coordinate of the 3-brane. The fields $X_{4,5}$ give rise to two gauge-singlet chiral multiplets (which we will denote $S_{1}$ and $S_{2}$ ).

There are several interesting things to note about this particle spectrum. First, the spectrum we found is manifestly not $N=2$ supersymmetric (since there is no chiral multiplet in the adjoint representation). Next, we expect that giving a vev to $A(B)$ corresponds to moving in the $z_{1}\left(z_{2}\right)$ direction, which should lead us to the $N=2$ theory. And indeed, giving a vev to $A(B)$ breaks the $S U(2) \times S U(2)$ gauge group to an $S U(2)$ subgroup, three components of $A(B)$ are swallowed by the Higgs mechanism, and we remain with an adjoint chiral multiplet (coming from $B(A)$ ) and additional singlets, as 
expected. It is less clear why an additional scalar appears from $X_{4,5}$ - from the analysis it is obvious that this additional scalar is massless only at the intersection point. The natural interpretation of this is that at the intersection point the 3-brane can split into two half-3-branes which can move independently (this is reasonable since at orientifold points the minimum quantum of RR charge is usually halved).

Next, we consider the 3-7 strings, namely, strings that stretch between the 3-brane and a 7-brane, giving rise to fields on the 3-brane world-volume field theory. The $\gamma_{\Omega}$ matrices for the 7-branes were computed in [24,25], and they are all proportional to the identity matrix. As shown in the appendix, one group of 7-branes (i.e. 7-branes located at a particular value of $z_{1}$ ) gives rise to eight chiral multiplets in the $(2,1)$ representation of the gauge group, which we will denote by $Q^{i}(i=1, \ldots, 8)$, while the other group (i.e. 7-branes located at a particular value of $z_{2}$ ) gives rise to eight chiral multiplets in the $(\mathbf{1}, 2)$ representation; those we will denote by $q^{i}(i=1, \ldots, 8)$.

Let us now analyze in detail the world-volume gauge theory at the orientifold point. Recall that we expect this theory to be an $N=1$ superconformal field theory, which should exist for any value of the gauge coupling (at least, any value of the $U(1)$ gauge coupling should be possible when we turn on small values of $z_{1}$ and $z_{2}$ ). Thus, we expect this theory to have a fixed line in the space of couplings, which passes through weak coupling.

The gauge group we found is $S U(2) \times S U(2)$. There are two chiral superfields in the $(2,2)$ representation ( $A$ and $B$ ), eight chiral superfields $Q$ in the $(2,1)$ representation and eight more chiral superfields $q$ in the $(1,2)$ representation (this matter content can be reinterpreted in terms of an $S O(4)$ gauge symmetry with two vectors and eight spinors). In the terminology of $N=1$, the theory has $N_{f}=6$ for each $S U$ (2) group factor and therefore, the one-loop beta function of the gauge coupling vanishes (as we would expect for a theory which has a fixed line passing through weak coupling).

Next, we should compute the superpotential of this theory. In principle, it is possible to compute this superpotential from the string theory analysis, but we have not performed this computation. Since we have only $N=1$ supersymmetry, the superpotential in general receives non-perturbative quantum corrections. However, we will try to guess what the superpotential should be by demanding that it reproduces our expectations of this theory. There are three main constraints on the superpotential:

(1) the theory should have (at least) an $S O(8) \times S O(8)$ global symmetry ( since this is the space-time gauge symmetry localized at the intersection point);

(2) the theory should flow to the $N=2, S U(2), N_{f}=4$ theory upon giving a vev to $A$ or to $B$;

(3) the theory should have a fixed line passing through weak coupling.

Note that at the orientifold point the 3-brane field theory is conformal, and terms of degree higher than 3 in the superpotential may also be important (as in Refs. [29,9]).

First, it is easy to see what the superpotential should be for the fields arising from $X_{4,5}$. The field $S_{1}$ corresponding to the location of the 3-brane in these coordinates should be decoupled, while the field $S_{2}$ (corresponding to splitting the 3-brane at the orientifold point) should be massless only when $A$ and $B$ are both zero. Thus, it is natural to guess 
a superpotential of the form $W=S_{2} A B .{ }^{9}$ With this superpotential, $A$ and $B$ become massive once $S_{2}$ is turned on, as expected since when the 3-brane has split we cannot move it away from the orientifold point.

The appearance of such a term in the superpotential is consistent also with the dimension we expect to find for the Coulomb branch of the theory (the phrase "Coulomb branch" here refers to a phase in which $A$ and $B$ may obtain vevs, but the quarks do not). We know that turning on $A^{2}$ and $B^{2}$ should correspond to flat directions of the field theory (when $S_{2}=0$ ), since the 3-brane should be free to move in the $z_{1}$ and $z_{2}$ directions, but these should be the only flat directions in the Coulomb phase. Thus, it should not be possible to turn on a vev for the gauge singlet $A B$, and this is exactly the effect of the superpotential $W=S_{2} A B$. This superpotential is also consistent with the flow to the $N=2$ theory upon turning on $A$ or $B$. If we give a vev to $A$, three of its components are swallowed by the Higgs mechanism, and the other remains massless and parametrizes the flat direction corresponding to the flow (it is the $N=2$ partner of $S_{1}$ ). Three of the components of $B$ remain massless and become an adjoint field $X$ of the remaining $S U(2)$, but the remaining component, as well as $S_{2}$, should become massive ( since we have no corresponding fields in the $N=2$ theory), and this is indeed the case if such a superpotential exists.

Finally, we should analyze which terms involving the quark field appear in the superpotential. The requirement of an $S O(8) \times S O(8)$ global symmetry (acting on the quarks in the obvious way), and of $S U(2) \times S U(2)$ gauge invariance, severely limits the possible terms that may appear. In fact, the only possible terms of degree four or less are $W=Q^{i} A B Q^{i}$ (which can be multiplied by some function $H\left(A^{2}, B^{2}\right)$ ) and $W=q^{i} A B q^{i}$ (which would then be multiplied by $H\left(B^{2}, A^{2}\right)$ ). When we give a vev to (say) $A$, these terms flow to the expected $N=2$ superpotential $q^{i} X q^{i}$, up to some function of $A^{2}$ and of $B^{2} \sim \operatorname{tr}\left(X^{2}\right)$. This function should equal 1 (or flow to 1 ) when $B=0$, so that for large $A$ and small $B, H\left(B^{2}, A^{2}\right)$ should behave like $1 / \sqrt{B^{2}}$.

The superpotential terms we have written fulfill several of our expectations, but there are two questions which we have not yet addressed that do not seem to be answered by these superpotentials. First, we expect the quarks $q^{i}\left(Q^{i}\right)$ to become massive when we give a vev to $A(B)$, so that we will flow to a theory with only eight quark doublets. Note that since we are now in an $N=1$ theory, there is no BPS mass formula from which we can compute the exact mass of these fields, but it seems obvious that they should become massive for non-zero $A(B)$. Unfortunately, it is not possible to write down an appropriate term in the superpotential that will preserve both the gauge symmetry and the global symmetry and that will give such a mass to the fields. For non-zero $A$ and $B$, we can write a superpotential of the form

$$
W=Q^{i} A B Q^{i} / \sqrt{A^{2}}+q^{i} A B q^{i} / \sqrt{B^{2}}
$$

that flows to the correct $N=2$ superpotential and gives the expected masses to the quarks. However, this superpotential is not well defined in the limit of $A \rightarrow 0$ (or $B \rightarrow 0$ ), and

\footnotetext{
${ }^{9}$ We thank A. Sen for this simplification to our original suggestion.
} 
there do not seem to be any other fields in the world-volume theory which would enable us to smooth this singularity.

A second problem is that we expect the theory to have a fixed line passing through weak coupling. To see the implications we follow, e.g., Ref. [29], where it is shown that for a theory with no scale dependence we need to require that the scaling coefficients

$$
\begin{gathered}
A_{g}=b_{1}+\sum_{i} T\left(R_{i}\right) \gamma_{i}, \\
A_{h_{n}}=(n-3)+\frac{1}{2} \sum_{k} \gamma_{k}
\end{gathered}
$$

vanish. Here, the sum in the scaling coefficient $A_{g}$ for the gauge couplings is over all fields in the theory, whereas the sum in $A_{h_{n}}$ is over all fields (with multiplicities) appearing in a degree- $n$ term in the superpotential; $h_{n}$ is the corresponding coupling constant and $b_{1}$ is the one-loop beta-function coefficient $b_{1}=3 C_{2}(G)-\sum_{i} T\left(R_{i}\right)$, which vanishes in our case. Having a fixed manifold of dimension higher than zero requires that the scaling coefficients are not all independent. In addition, having a fixed line through weak coupling, where the anomalous dimensions are expected to vanish, requires $d_{n}=3$. However, in our case it is impossible to write simple polynomial potentials of degree 3 , but only superpotentials of the form (3.8), whose interpretation is not clear. Note that if we use the superpotential (3.8), the equations in (3.9) are linearly dependent, so a fixed line (passing through weak coupling) is expected to exist, but we do not understand how such a superpotential may arise.

As in [9], we can compute the dimensions of some of the operators of the fixed-point theory from the string theory metric. The elliptic curve describing the theory near the fixed point looks like $y^{2} \sim x^{3}+z_{1}^{2} z_{2}^{2} x+z_{1}^{3} z_{2}^{3}$, so that $\left[z_{1}\right]+\left[z_{2}\right]=[x]$, and the equation $\left[z_{1}\right]+\left[z_{2}\right]+[x]-[y]=2[9]$ leads to $\left[z_{1}\right]+\left[z_{2}\right]=4$, so that $[A]+[B]=2$ and it is natural to assume that $[A]=[B]=1$, so that both fields have no anomalous dimension. This is consistent with having a fixed line that goes through weak coupling along which the dimensions do not change, as was the case for the $N=2$ theory (and for the $N=1$ theory discussed in [9]). If $A$ and $B$ have no anomalous dimensions, then the requirement of the vanishing of the beta function (3.9) leads to $Q$ and $q$ having no anomalous dimensions either. Then, it is clear that, even if we do not demand that the fixed line passes through weak coupling, only dimension-3 superpotentials could be relevant at the fixed point. Again, this is consistent with using the superpotential (3.8) whose interpretation is not clear, but not, as far as we could see, with any other superpotentials.

To summarize, we have computed by a perturbative string theory analysis the spectrum of the 3-brane probe world-volume theory near an intersection of two $\mathrm{D}_{4}$ singularities in a particular global context (i.e. an orientifold which is equivalent, up to discrete torsion, to F-theory compactified on $\left.\mathbf{T}^{6} /\left(\mathbb{Z}_{2} \times \mathbb{Z}_{2}\right)\right)$. The field content we have found is consistent with our expectations, but we have not been able to write down a superpotential at the intersection point that will reproduce all of our expectations (although we can write a 
superpotential away from the orientifold points that does seem to be consistent). It seems that the field theory at the fixed point cannot be described by a simple (i.e. polynomial) superpotential. This could be related to the fact that the theory has massless magnetic and dyonic degrees of freedom as well as electric. However, in the $N=2$ case that did not prevent a simple superpotential description from existing (of course, the supersymmetry constraints were much stronger in that case). Another possibility, which seems less likely, is that in the case we are discussing the fields corresponding to the other orientifold points no longer decouple, and must all be included in the analysis. A third possibility is that our problems are related to the existence of a tensor multiplet in the space-time theory the probe couples to at the intersection point. In principle, we should be able to include in our field theory the coupling to the space-time tensor multiplet as well. The scalar in this multiplet should correspond to a real scalar parameter of the 3-brane field theory. Turning on this scalar corresponds to blowing the point $z_{1}=z_{2}=0$ into a 2-cycle, after which the two $D_{4}$ singularities no longer meet. In the field theory the moduli space should change in a similar way, and the origin of moduli space should be blown up. There should no longer be a point of unbroken $S U(2) \times S U(2)$, but only non-intersecting lines of unbroken $S U(2)$. Obviously, the appropriate terms are in the Kähler potential and not in the superpotential, and we have not analyzed the exact form that they should take. The effect of the space-time 2-form field on the world-volume theory is far less clear.

Another interesting question is the relation between the field theory we discuss, involving the 3-brane in the orientifold background, and the field theory describing a 3-brane moving in the F-theory background with no 2-form fields turned on. We have not identified what the discrete torsion which differentiates between the two theories corresponds to in the 3-brane field theory, so the relation between these field theories is not clear. As discussed in the next section, the discrete torsion definitely changes the field theory at the origin of moduli space, although (since the discrete torsion is concentrated at the origin) it does not change the Coulomb branch away from the origin. Thus, the 3-brane moving in the F-theory background should be described by a different field theory (which should have exact $S L(2, \mathbb{Z})$ electric-magnetic duality), but the Coulomb phase of all these theories should be identical. In the F-theory background, tensionless strings appear in space-time, and the effect these should have on the 3-brane probe field theory is also unknown.

We can easily generalize the analysis of this section to multiple 3-brane probes, as in Section 2. We find for $k$ 3-brane probes an $S p(2 k) \times S p(2 k)$ gauge theory, with two chiral superfields in the $(\mathbf{2 k}, \mathbf{2 k})$ representation from $X_{6,7,8,9}$, superfields in the $(\mathbf{k}(2 \mathbf{k}-\mathbf{1})-\mathbf{1}, \mathbf{1})+(\mathbf{1}, \mathbf{1})+(\mathbf{1}, \mathbf{k}(\mathbf{2 k}-\mathbf{1})-\mathbf{1}, \mathbf{1})+(\mathbf{1}, \mathbf{1})$ representations from $X_{4,5}$, and eight $(2 \mathbf{k}, \mathbf{1})$ and $(\mathbf{1}, \mathbf{2 k})$ superfields from the 3-7 strings. The Coulomb phase of this theory may be easily seen to consist of $k$ copies of the Coulomb phase of the original theory (up to an $S_{k}$ identification), and we run into the same problems when trying to write a superpotential describing the theory at the origin of the moduli space.

At other types of intersection points we also expect to find $N=1$ superconformal theories with various global symmetries. For instance, at the intersection of two $E_{n}$ singularities, we should find an $N=1$ superconformal theory with $E_{n} \times E_{n}$ global 
symmetry. As in [9], we can compute the dimensions of some of the operators in these $N=1$ SCFTs, but so far we do not have a lagrangian that would flow to them, hence we cannot say much more about them.

\section{Summary and open questions}

In this paper we discussed two field theories arising from 3-brane probes in F-theory. In Section 2 we discussed the field theory corresponding to $k$ parallel 3-brane probes in F-theory compactified on $\mathbf{T}^{4} / \mathbb{Z}_{2}$. As expected from string theory, we found that the Coulomb branch of the corresponding field theory was equivalent to $k$ copies of the Coulomb branch of the original $k=1$ theory, divided by $S_{k}$. We expect this to be the general result for multiple $D$-brane probes.

In Section 3 we discussed a 3-brane probe at an intersection of two $D_{4}$ singularities, in a particular orientifold vacuum of string theory. By general considerations we expect this to correspond to an $N=1$ superconformal field theory, which can flow (in two different ways) to the Seiberg-Witten $N=2 S U(2) N_{f}=4$ theory. The field content we found on the probe was consistent with this expectation, but we have not been able to write down an explicit superpotential that would be consistent with everything we know. Specifically, we were not able to write down a superpotential that would give masses to some of the quarks when we flow to the $N=2$ theory, and that would naturally lead to the existence of a fixed line in the field-theory moduli space. We are not sure about the meaning of this failure. It might be related to the interaction of the probe with a tensor multiplet in space-time, which may prevent us from having a simple local description. It would be very interesting to understand the resolution of this problem, which could also be related to the problems encountered in the space-time description of intersecting singularities.

Next, we would like to discuss is the correspondence between the space-time fields and the parameters on the D-brane probe theory. It is clear that any space-time field should correspond to a parameter in the world-volume theory, since the D-brane couples in a consistent way to the space-time theory. However, a relation in the opposite direction does not necessarily have to exist. In principle, there could exist parameters of the worldvolume theory that do not correspond to any space-time fields, and the corresponding theories would not be realized in the moduli space of a D-brane moving in the string theory vacuum. One example of this phenomenon is the theory we discuss in Section 2, where a mass term for the antisymmetric $S p(2 k)$ field is allowed in the field theory, but the corresponding parameter does not exist in string theory (as noted also in [30]).

Another example involves the quark masses in the theory we discuss in Section 3. In eight dimensions, there was $[1,2]$ an exact correspondence between the deformations of the space-time theory and the ( $N=2$ preserving) parameters of the world-volume theory. In particular, the $S O(8)$ adjoint scalars in space-time (which were part of the vector multiplet), corresponding to moving the 7-branes off the orientifold, were interpreted as a mass matrix for the quarks in the world-volume theory. In the $6 \mathrm{D}$ generalization we considered in Section 3 the situation is different and more complicated, as we will now 
explain. The orientifold we consider, corresponding to a compactification of F-theory on $\mathbf{T}^{6} / \mathbb{Z}_{2} \times \mathbb{Z}_{2}$, is not connected in any simple way to the elliptic fibration over $\mathbf{F}_{0}$. Instead, the orientifold compactification is connected to a compactification of F-theory on a Calabi-Yau manifold with Hodge numbers $(51,3)$ [24-26]. In this case the orientifold analysis reveals that in 6D space-time we do not have any charged fields. Thus, we cannot break the $S O(8)$ gauge symmetry by the appropriate Higgsing and, therefore, we do not get quark mass parameters on the 3-brane world-volume theory. Note that at the orientifold point we have altogether 17 tensor multiplets and four hypermultiplets. This field content is consistent with the cancelation of $6 \mathrm{D}$ anomalies. ${ }^{10}$ The tensor multiplet localized at each intersection point of groups of seven-branes contains a scalar field, which provides a possible deformation of the theory. However, this deformation does not resolve the $D_{4}$ singularities, but just separates the two intersecting singularities [23]. The four hypermultiplets in space-time correspond to moduli of the compactification manifold, and do not affect our analysis near a single orientifold point. Thus, the moduli of the orientifold theory do not include fields corresponding to quark mass matrices in this case. To get such fields, we should go through phase transitions which would turn the 16 extra tensor multiplets into hypermultiplets, taking us to the general curve in Weierstrass form [22] which has many (243) parameters. The modulus of the F-theory torus, $\tau$, will, generically, depend on all these parameters. Some of these parameters will presumably correspond to quark mass matrices. It is a challenging problem to understand this phase transition (which is similar to the M-theory phase transition in which a 5-brane (tensor) is "swallowed" by a 9-brane and turns into a large $E_{8}$ instanton) and its effects on the 3-brane world-volume field theory.

Finally, we now present a brief analysis of the model of Sen [11] along the lines of Section 3. ${ }^{11}$ But before doing that, we want to point out some of the differences of the two models; for details we refer to [24-26]. Both models can be described as type IIB on $\mathrm{K} 3$ orientifolds. They differ, however, in the action of the orientifold projections on the twisted sector and open string states. The model considered by Sen [11] is $T$-dual to the Gimon-Polchinski model, and thus does not contain any extra tensor multiplets, while the model we have considered has extra tensor multiplets and is clearly different (it has been constructed explicitly in [24-26]). From the F-theory point of view, it seems that both models correspond to the same elliptic curve (3.1)-(3.3), but they differ by discrete 2 -form fields concentrated at the intersection of the singularities (as in the analogous string theory compactifications discussed in [27]). The two models both have such discrete torsion turned on, so that none of them correspond to the naive F-theory compactification, but the values of the discrete 2 -form fields are different in the two cases. In both cases the discrete 2-form fields prevent the 3-brane from wrapping the collapsed 2 -cycle and giving rise to tensionless strings. In Sen's model the low-energy spectrum

\footnotetext{
${ }^{10}$ Note that the disappearance of the $S O(8)$ adjoint field in the orientifold procedure is consistent with the anomaly equation for $\operatorname{tr}\left(F^{4}\right)$, due to the relation $\operatorname{tr}_{28} F^{4}=3\left(\operatorname{tr}_{8} F^{2}\right)^{2}$ (without any $\operatorname{tr}_{8} F^{4}$ term).

${ }^{11}$ This is an extended version of a note added to our original preprint version. It was sparked by remarks of A. Sen, who also suggested that for his model there might be just the right field content on the probe to satisfy all the conditions we have required for the superpotentials. This is what we are going to show here.
} 
in space-time is also different from the naive F-theory spectrum, suggesting that in this case both 2-form fields are turned on, while in the orientifold we discussed in Section 3 apparently only one of the 2-form fields is turned on. As before, our computation here will be in the orientifold theory, and its direct relation to F-theory is not clear.

It is straightforward to repeat the analysis which leads to the spectrum on the 3-brane probe for the model considered by Sen. There is no change in our discussion of the states arising from the 3-3 strings. Since the model considered by Sen is just the $T$-dual of the model studied in detail by Gimon and Polchinski [12] - the $T$-duality acting, say, in the 89 plane - we can immediately read off the matrices $\gamma$ acting on the 7-brane indices of states in the $7-7,7-7^{\prime}, 7^{\prime}-7^{\prime}$ sectors and in the $3-7$ and $3-7^{\prime}$ sectors, from the ones given in [12], by $T$-dualizing. One finds that

$$
\begin{gathered}
\gamma_{\Omega_{1}}^{(7)}=\gamma_{\Omega_{2}}^{\left(7^{\prime}\right)}=\mathbf{1}_{8 \times 8}, \\
\gamma_{T}^{(7)}=\gamma_{T}^{\left(7^{\prime}\right)}=\gamma_{\Omega_{2}}^{(7)}=\gamma_{\Omega_{1}}^{\left(7^{\prime}\right)}=\left(\begin{array}{cc}
0 & i \mathbf{1}_{4 \times 4} \\
-i \mathbf{1}_{4 \times 4} & 0
\end{array}\right) .
\end{gathered}
$$

Note that the $\gamma^{(7)}$ and $\gamma^{\left(7^{\prime}\right)}$ matrices are not all proportional to the identity matrix, reflecting the fact that the space-time gauge symmetry is no longer $S O(8)$, but in fact the subgroup $U(4)$. The global symmetry of the probe theory is thus $U(4) \times U(4)$ instead of $S O(8) \times S O(8)$; see also Ref. [11]. This is part of the $U(4)^{8}$ gauge symmetry of the space-time theory $[12,11]$. Also, from [12] we learn that in the space-time theory there are, in addition to the gravity multiplet, the $U(4) \times U(4)$ gauge fields and one anti-self-dual tensor multiplet, also massless hypermultiplets: $2(6,1)+2(1,6)+(4,4)$ (which will correspond to mass terms in our probe theory). Redoing our analysis of the 3-7 and 3-7' states with the new 7-brane gamma matrices, we find states in the following representations of the local and global symmetry group $S U(2) \times S U(2) \times U(4) \times U(4)$ : $(2,1,4,1)+(1,2, \overline{4}, 1)+(2,1,1,4)+(1,2,1, \overline{4})$. In addition to these chiral multiplets, we still have, as before, the chiral superfields $A, B$ from the 3-3 strings, both transforming as $(2,2,1,1)$. Given the field content of this probe theory, we can now easily write down a superpotential which satisfies all the conditions described in Section 3. This probe theory thus provides the question to which Sen [11] has given the string theory answer. Denoting $(\mathbf{2}, \mathbf{1}, \mathbf{4}, \mathbf{1}) \hat{=} Q,(\mathbf{1}, \mathbf{2}, \overline{\mathbf{4}}, \mathbf{1}) \hat{=} \tilde{Q},(\mathbf{2}, \mathbf{1}, \mathbf{1}, \mathbf{4}) \hat{=} q,(\mathbf{1}, \mathbf{2}, \mathbf{1}, \overline{\mathbf{4}}) \hat{=} \tilde{q}$ one can now write a superpotential of the form $W=Q A \tilde{Q}+q B \tilde{q}$ so that the conditions of Ref. [29] for having a fixed line passing through the origin of the space of couplings are fulfilled. This provides an explicit realization of an $N=1$ supersymmetric theory which has an exact electric-magnetic duality, as is evident from the string theory answer. The duality group in this case, due to the presence of the discrete torsion, is the $\Gamma(2)$ subgroup of $S L(2, \mathbb{Z})$. Turning on an expectation value for $A(B)$ gives masses to the $Q, \tilde{Q}(q, \tilde{q})$ quarks, and the theory flows to the $N=2 S U(2)_{D}$ theory of [1]. Recall that $B$ transforms under the unbroken $S U(2)_{D}$ as $\mathbf{3}+\mathbf{1}$ so that a superpotential of the form $q X \tilde{q}$, where $X$ is in the adjoint of $S U(2)_{D}$, emerges form the $q B \tilde{q}$ term.

We are tempted to conjecture that the difficulties that we encountered in our original model, which are absent in the model considered by Sen, are related to the fact that in 
the former we have extra tensor multiplets which are absent in the latter. As discussed above, the two models seem to differ only by discrete 2-form fields concentrated at the singularities. Since these 2 -form fields can assume only discrete values, one cannot relate the two theories simply by changing these fields continuously. It would be interesting to understand if the theories could be related by some sort of phase transition. Such a phase transition would obviously need to transform the 16 extra tensor multiplets in the first orientifold we analyzed into hypermultiplets in the second orientifold (similar to the phase transition related to the small $E_{8}$ instanton). This issue deserves further investigation.

\section{Acknowledgements}

We would like to thank P. Aspinwall, S. Kachru, P. Mayr, R. Plesser, N. Seiberg, E. Silverstein and E. Witten for useful discussions. We thank, in particular, V. Kaplunovsky for comments and for sharing with us his insight on issues related to Section 2. Special thanks also to A. Sen for communications after the first version of this paper appeared. The work of O.A. was supported in part by DOE grant DE-FG02-96ER40559.

\section{Appendix A. Explicit determination of the representations of the vector and chiral superfields}

\section{A.1. Fields from the 3-brane strings}

First we determine the fields that correspond to open strings with both ends on the 3-brane (or on its images). In flat space the 3-brane field theory involves an $N=4$ vector multiplet, containing an $N=1$ vector multiplet and three $N=1$ chiral multiplets corresponding to the coordinates $X_{4,5,6,7,8,9}$ of the 3 -brane. In the presence of the orientifold, each of these fields is enhanced into a $4 \times 4$ matrix with different constraints.

\section{Vector superfields}

The relations imposed by (3.7) on the components $M_{i j}$ of gauge fields are

$$
\begin{aligned}
& M_{11}=-M_{22}=-M_{33}=M_{44}, \\
& M_{14}=M_{41}=M_{23}=M_{32}, \\
& M_{12}=-M_{43}, \quad M_{13}=-M_{42}, \\
& M_{21}=-M_{34}, \quad M_{31}=-M_{24} .
\end{aligned}
$$

A basis of six matrices that obey these relations is 


$$
\begin{array}{rlrl}
Z_{1}= & \left(\begin{array}{cccc}
1 & 0 & 0 & 0 \\
0 & -1 & 0 & 0 \\
0 & 0 & -1 & 0 \\
0 & 0 & 0 & 1
\end{array}\right), & Z_{2} & =\left(\begin{array}{cccc}
0 & 0 & 0 & -1 \\
0 & 0 & -1 & 0 \\
0 & -1 & 0 & 0 \\
-1 & 0 & 0 & 0
\end{array}\right) \\
W_{1}=\left(\begin{array}{cccc}
0 & 1 & 0 & 0 \\
1 & 0 & 0 & 0 \\
0 & 0 & 0 & -1 \\
0 & 0 & -1 & 0
\end{array}\right), & W_{2}=\left(\begin{array}{cccc}
0 & 0 & 1 & 0 \\
0 & 0 & 0 & -1 \\
1 & 0 & 0 & 0 \\
0 & -1 & 0 & 0
\end{array}\right) \\
W_{3}=\left(\begin{array}{cccc}
0 & 0 & i & 0 \\
0 & 0 & 0 & i \\
-i & 0 & 0 & 0 \\
0 & -i & 0 & 0
\end{array}\right), & W_{4}=\left(\begin{array}{cccc}
0 & i & 0 & 0 \\
-i & 0 & 0 & 0 \\
0 & 0 & 0 & i \\
0 & 0 & -i & 0
\end{array}\right) .
\end{array}
$$

It is now straightforward to check that the matrices

$$
\begin{aligned}
& W_{2}^{+}=\frac{1}{4}\left(W_{1}+W_{2}\right), \quad W_{1}^{-}=\frac{1}{4}\left(W_{1}-W_{2}\right), \quad W_{1}^{+}=\frac{1}{4}\left(W_{3}+W_{4}\right), \\
& W_{2}^{-}=\frac{1}{4}\left(W_{3}-W_{4}\right), \quad Z^{ \pm}=\frac{1}{4}\left(Z_{1} \pm Z_{2}\right)
\end{aligned}
$$

obey the following commutation relations:

$$
\begin{array}{rlrl}
{\left[Z^{+}, W_{2}^{+}\right]} & =-i W_{1}^{+}, & {\left[Z^{+}, W_{1}^{+}\right]=i W_{2}^{+}, \quad\left[W_{2}^{+}, W_{1}^{+}\right]=-i Z^{+},} \\
{\left[Z^{-}, W_{1}^{-}\right]} & =i W_{2}^{-}, \quad\left[Z^{-}, W_{2}^{-}\right]=-i W_{1}^{-}, \quad\left[W_{1}^{-}, W_{2}^{-}\right]=i Z^{-}, \\
{\left[Z^{+}, W_{1}^{-}\right]} & =0, \quad\left[Z^{+}, W_{2}^{-}\right]=0, \quad\left[Z^{-}, W_{2}^{+}\right]=0, \quad\left[Z^{-}, W_{1}^{+}\right]=0, \\
{\left[Z^{+}, Z^{-}\right]} & =0, & {\left[W_{2}^{+}, W_{1}^{-}\right]=0,} & {\left[W_{2}^{+}, W_{2}^{-}\right]=0,} \\
{\left[W_{1}^{+}, W_{1}^{-}\right]} & =0, & {\left[W_{1}^{+}, W_{2}^{-}\right]=0 .}
\end{array}
$$

Thus, we see that the gauge fields span an $S U(2) \times S U(2)$ algebra. When we take $z_{1}$ to infinity all $1-2,1-4,2-3$ and 3-4 strings become infinitely massive, so we can drop those wave-function matrices with non-zero entries in those positions. This leaves $Z_{1}, W_{2}$ and $W_{3}$, which generate an $S U(2)$ subalgebra. Likewise, if we take $z_{2}$ to infinity all 1-3, 1-4, 2-3 and 2-4 strings become massive, and we are left with $Z_{1}, W_{1}$ and $W_{4}$, which generate a different $S U(2)$ subalgebra (which does not commute with the previous one). This picture is in accord with the naive expectation following Ref. [1] of having just a single $S U(2)$ near $z_{1}=0$ or $z_{2}=0$.

\section{$X_{67}$ chiral multiplet}

Next consider the implications of (3.7) on the scalar fields $X_{67}$. The constraints on the matrix components are now

$$
\begin{aligned}
& M_{11}=-M_{22}=M_{33}=-M_{44}, \\
& M_{14}=-M_{41}=-M_{23}=M_{32}, \\
& M_{12}=+M_{43}, \quad M_{13}=M_{42}=0,
\end{aligned}
$$




$$
M_{21}=+M_{34}, \quad M_{31}=M_{24}=0 .
$$

A basis of hermitian matrices that obey the relations is the following

$$
\begin{aligned}
A_{1} & =\left(\begin{array}{cccc}
1 & 0 & 0 & 0 \\
0 & -1 & 0 & 0 \\
0 & 0 & 1 & 0 \\
0 & 0 & 0 & -1
\end{array}\right), & A_{2} & =\left(\begin{array}{cccc}
0 & 0 & 0 & i \\
0 & 0 & -i & 0 \\
0 & i & 0 & 0 \\
-i & 0 & 0 & 0
\end{array}\right), \\
A_{3} & =\left(\begin{array}{cccc}
0 & 1 & 0 & 0 \\
1 & 0 & 0 & 0 \\
0 & 0 & 0 & 1 \\
0 & 0 & 1 & 0
\end{array}\right), & A_{4} & =\left(\begin{array}{cccc}
0 & i & 0 & 0 \\
-i & 0 & 0 & 0 \\
0 & 0 & 0 & -i \\
0 & 0 & i & 0
\end{array}\right) .
\end{aligned}
$$

Note that now if we take $z_{1}$ to infinity (naively) we are left only with $A_{1}$, while if we take $z_{2}$ to infinity (naively) we remain with $A_{1}, A_{3}$ and $A_{4}$ which are in the adjoint representation of the remaining $S U(2)$, and thus we go over to the picture of [1], as expected.

Define now the matrices $A_{-+}, A_{++}, A_{--}$and $A_{+-}$by the equations $A_{++}=A_{3}-i A_{4}$; $A_{-+}=A_{1}+i A_{2} ; A_{+-}=A_{1}-i A_{2}$ and $A_{--}=A_{3}+i A_{4}$. It can easily be checked that $A=\left(A_{++}, A_{-+}, A_{+-}, A_{--}\right)$are in the $(2,2)$ representation of $S U(2) \times S U(2)$, with the subscripts corresponding to the charges under $Z^{+}$and $Z^{-}$(note that these matrices are not hermitian). For instance, we have

$$
\begin{array}{ll}
{\left[Z^{+}, A_{++}\right]=\frac{1}{2} A_{++},} & {\left[Z^{+}, A_{-+}\right]=-\frac{1}{2} A_{-+},} \\
{\left[W_{2}^{+}, A_{++}\right]=-\frac{1}{2} A_{-+},} & {\left[W_{2}^{+}, A_{-+}\right]=-\frac{1}{2} A_{++},} \\
{\left[W_{1}^{+}, A_{++}\right]=\frac{i}{2} A_{-+},} & {\left[W_{1}^{+}, A_{-+}\right]=-\frac{i}{2} A_{++}}
\end{array}
$$

and so on.

\section{$X_{89}$ chiral multiplet}

For the relations on the $X_{89}$ chiral superfield we would find similar (but not identical) results to those of the previous section, with the second and third rows and columns of all matrices interchanged. Thus, this chiral superfield, that we denote by $B=\left(B_{++}, B_{-+}, B_{+-}, B_{--}\right)$, is also in the $(2,2)$ representation of $S U(2) \times S U(2)$ (though they are not represented in the same way).

As a consistency check, we verify that a vev for $X_{67}$, for instance, indeed breaks the gauge symmetry to a diagonal $S U(2)$. For instance, taking $A_{1}$ to have a vev would leave exactly the matrices $Z_{1}, W_{2}$ and $W_{3}$ (given above), which commute with it, as expected. The $A$ 's would also all become massive except $A_{1}$ (since they do not commute with it), again as expected (since after moving along the flat direction we should have just a single scalar). Vevs for both $X_{67}$ and $X_{89}$ can also be analyzed and they all behave as expected from naive considerations. 


\section{$X_{45}$ chiral multiplet}

The relations for $X_{45}$ are

$$
\begin{aligned}
& M_{11}=M_{22}=M_{33}=M_{44}, \\
& M_{14}=M_{41}=-M_{23}=-M_{32},
\end{aligned}
$$

and their solutions are spanned by the two singlets $S_{1}$ and $S_{2}$,

$$
S_{1}=\left(\begin{array}{cccc}
1 & 0 & 0 & 0 \\
0 & 1 & 0 & 0 \\
0 & 0 & 1 & 0 \\
0 & 0 & 0 & 1
\end{array}\right), \quad S_{2}=\left(\begin{array}{cccc}
0 & 0 & 0 & 1 \\
0 & 0 & -1 & 0 \\
0 & -1 & 0 & 0 \\
1 & 0 & 0 & 0
\end{array}\right)
$$

\section{A.2. Fields form the strings between the 3-brane and 7-branes}

As mentioned in Section 3, the $\gamma_{\Omega}$ matrices for the 7-branes are all proportional to the identity matrix [24-26]. The constraint on the 7-brane gauge fields is then just $M=-M^{T}$, giving an anti-symmetric matrix corresponding to an $S O(8)$ space-time gauge symmetry, since there are eight 7 -branes when including the $Z_{2}$ partners.

Next, we should use these matrices to analyze the wave function of the 3-7 strings (as in [12]). The 7-brane side is trivial, so the orbifold/orientifold projections just mix the various 3-branes according to the $\gamma_{\Omega}$ matrices given in (3.7); for instance taking the $3_{1}-7$ state (where $3_{1}$ is the first 3-brane) via $\gamma_{\Omega_{1}}$ to $i$ times the 7-3 state (with opposite orientation!), via $\gamma_{\Omega_{2}}$ to $i$ times the $7-3_{3}$ state, and via $\gamma_{T}$ to minus the $3_{4}-7$ state.

A basis for the states going to a specific 7-brane can thus be chosen to be

$$
D_{u}^{+}=(1,0,0,-1), \quad D_{d}^{+}=(0,1,1,0) .
$$

Using the matrices we found above for the gauge fields, it is easy to check that these are doublets under $S U(2)_{+}$, and singlets with respect to $S U(2)_{-}$. The corresponding chiral superfields are thus in a $(2,1)$ representation of $S U(2)_{+} \times S U(2) \ldots$ For the other group of 7-branes we can then do the same thing, but with minus the identity matrix for some of the relevant 7-7 $\gamma_{\Omega}$ matrices (say for $\gamma_{\Omega_{2}}$ and $\gamma_{T}$ ). Then, the basis comes out to be

$$
D_{u}^{-}=(1,0,0,1), \quad D_{d}^{-}=(0,1,-1,0) .
$$

which is in the $(\mathbf{1}, \mathbf{2})$ representation of $S U(2)_{+} \times S U(2)_{-}$.

\section{References}

[1] A. Sen, F-Theory and Orientifolds, Nucl. Phys. B 475 (1996) 562, hep-th/9605150.

[2] T. Banks, M.R. Douglas and N. Seiberg, Probing F-Theory With Branes, Phys. Lett. B 387 (1996) 278 , hep-th/9605199.

[3] C. Vafa, Evidence for F-Theory, Nucl. Phys. B 469 (1996) 403, hep-th/9602022.

[4] N. Seiberg and E. Witten, Electric-Magnetic Duality, Monopole Condensation and Confinement in $N=2$ Supersymmetric Yang-Mills Theory, Nucl. Phys. B 426 (1994) 19, hep-th/9407087. 
[5] N. Seiberg and E. Witten, Monopoles, Duality and Chiral Symmetry Breaking in $N=2$ Supersymmetric QCD, Nucl. Phys. B 431 (1994) 484, hep-th/9408099.

[6] M.R. Douglas, Branes within Branes, hep-th/9512077;

M.R. Douglas and G. Moore, D-branes, Quivers, and ALE Instantons, hep-th/9603167;

M.R. Douglas, Gauge Fields and D-branes, hep-th/9604198.

[7] N. Seiberg, IR Dynamics on Branes and Space-time Geometry, Phys. Lett. B 384 (1996) 81, hepth/9606017.

[8] N. Seiberg, Five-Dimensional SUSY Field Theories, Non-trivial Fixed Points and String Dynamics, Phys. Lett. B 388 (1996) 753, hep-th/9608111.

[9] O. Aharony, S. Kachru and E. Silverstein, New $N=1$ superconformal field theories in four dimensions from D-brane probes, hep-th/9610205.

[ I0] O.J. Ganor, D.R. Morrison and N. Seiberg, Branes, Calabi-Yau Spaces, and Toroidal Compactification of the N=1 Six-Dimensional $E_{8}$ Theory, Nucl. Phys. B 487 (1997) 93, hep-th/9610251.

[11] A. Sen, A Non-perturbative Description of the Gimon-Polchinski Orientifold, hep-th/9611186.

[12] E.G. Gimon and J. Polchinski, Consistency Conditions for Orientifolds and D-Manifolds, Phys. Rev. D 54 (1996) 1667, hep-th/9601038.

[13] J. Polchinski, Dirichlet Branes and Ramond-Ramond Charges, Phys. Rev. Lett. 75 (1995) 4724, hep-th/9510169.

[14] E. Witten, Small Instantons in String Theory, Nucl. Phys. B 460 (1996) 541, hep-th/9511030.

[15] P.C. Argyres and A.D. Shapere, The Vacuum Structure of $N=2$ SuperQCD with Classical Gauge Groups, Nucl. Phys. B 461 (1996) 437, hep-th/9509175.

[16] E. Witten, Bound States of Strings and p-branes, Nucl. Phys. B 460 (1996) 335, hep-th/9510135.

[17] R. Donagi and E. Witten, Supersymmetric Yang-Mills Theory and Integrable Systems, Nucl. Phys. B 460 (1996) 299, hep-th/9510101.

[18] P.C. Argyres, M.R. Plesser, N. Seiberg and E. Witten, New $N=2$ superconformal field theories in four dimensions, Nucl. Phys. B 461 (1996) 71, hep-th/9511154.

[19] J. Minahan and D. Nemeschansky, An $\mathcal{N}=2$ Superconformal Fixed Point with $E_{6}$ Global Symmetry, Nucl. Phys. B 482 (1996) 142, hep-th/9608047;

W. Lerche and N. Warner, Exceptional SW Geometry from ALE Fibrations, hep-th/9608183;

J. Minahan and D. Nemeschansky, Superconformal Fixed Points with $E_{n}$ Global Symmetry, hepth/9610076.

[20] O.J. Ganor, Toroidal Compactification of Heterotic 6D Non-critical Strings Down to Four Dimensions, hep-th/9608109.

[21] M. Bershadsky, K. Intriligator, S. Kachru, D.R. Morrison, V. Sadov and C. Vafa, Geometric Singularities and Enhanced Gauge Symmetries, Nucl. Phys. B 481 (1996) 215, hep-th/9605200.

[22] D.R. Morrison and C. Vafa, Compactifications of F-Theory on Calabi-Yau Threefolds I, Nucl. Phys. B 473 (1996) 74, hep-th/9602114; Compactifications of F-Theory on Calabi-Yau Threefolds II, Nucl. Phys. B 476 (1996) 437, hep-th/9603161.

[23] M. Bershadsky and A. Johansen, Colliding Singularities in F Theory and Phase Transitions, hepth/9610111.

[24] J.D. Blum and A. Zaffaroni, An Orientifold from F-Theory, Phys. Lett. B 387 (1996) 71, hepth/9607019.

[25] A. Dabholkar and J. Park, A Note on Orientifolds and F-Theory, hep-th/9607041.

[26] R. Gopakumar and S. Mukhi, Orbifold and Orientifold Compactifications of F-Theory and M Theory to Six and Four Dimensions, Nucl. Phys. B 479 (1996) 260, hep-th/9607057.

[27] C. Vafa and E. Witten, On Orbifolds With Discrete Torsion, J. Geom. Phys. 15 (1995) 189, hepth/9409188.

[28] P. Aspinwall, Enhanced Gauge Symmetries and K3 Surfaces, Phys. Lett. B 357 (1995) 329, hepth/9507012.

[29] R.G. Leigh and M. Strassler, Exactly Marginal Operators and Duality in Four Dimensional $N=1$ Supersymmetric Gauge Theory, Nucl. Phys. B 447 (1995) 95, hep-th/9503121.

[30] J. de Boer, K. Hori, H. Ooguri and Y. Oz, Mirror Symmetry in Three-Dimensional Gauge Theories, Quivers and D-branes, Nucl. Phys. B (1997), to be published, hep-th/9611063. 